\title{
Harvesting analysis of a discrete competitive system
}

\section{Ting $W^{*}$}

\section{${ }^{*}$ Correspondence:}

wuting723@tom.com;

wuting369@tom.com

Department of Mathematics,

Minjiang University, Fuzhou, Fujian 350002, P.R. China

\begin{abstract}
In this paper, we discuss a discrete competitive system based on density dependence to obtain a set of sufficient conditions for the existence and asymptotic stability of the equilibrium of systems. By obtaining the optimal harvest strategy of systems through the extreme value method and the discrete Pontryagin maximum principle, we provide a theoretical direction for the actual production.
\end{abstract}

Keywords: discrete; competitive systems; equilibrium; global stability; fishing companies; profit

\section{Introduction}

Stability and permanence of a biological system have been studied by several authors [14]. The problem of fractional differential equation was also studied [5-13]. However, the rational development and management of the biological resources were directly related to sustainable development. In recent years, continuous system capture has received many scholars' attention [14-22]. Similarly, the optimal control theory is a good method [2327]. In fact, as we know, fish distribution is inhomogeneous and it is not possible to capture successively. Therefore, it is more reasonable to consider the discrete system's capture. Not only it will keep the biological balance but it will also save time and produce more economic revenue for fishermen. Due to the peculiarity of the discrete system, it is difficult to study its stability and capture, and there are few related studies. Therefore, in this paper, we consider the following discrete two species competitive system and discuss the system's stability and capturing strategy,

$$
\left\{\begin{array}{l}
\Delta x_{n}=x_{n}\left(a_{1}-b_{1} x_{n}-c_{1} y_{n}\right)-E_{1} x_{n}=P_{1}\left(x_{n}, y_{n}\right), \\
\Delta y_{n}=y_{n}\left(a_{2}-b_{2} x_{n}-c_{2} y_{n}\right)-E_{2} y_{n}=P_{2}\left(x_{n}, y_{n}\right) .
\end{array}\right.
$$

Here $a_{1}$ and $a_{2}\left(a_{1}>0, a_{2}>0\right)$ denote the intrinsic growth rate of two species $x_{n}$ and $y_{n}$ (or life factor). $b_{1}$ and $c_{2}\left(b_{1}, c_{2}>0\right)$ denote the density-dependent entry. Generally speaking, two populations are both caught by fishermen. It has practical significance to take the capture effect into consideration in order to reap the maximum economic benefits. Let $E_{1}, E_{2}$ $\left(E_{1}, E_{2}>0\right)$ be the capture intensity of the two populations (that is, fishing effort multiplies the capture coefficients) $\left(E_{1}+E_{2}=E\right)$, and let the capture per unit time be proportional to the stock and population, $a_{1}>E_{1}$ and $a_{2}>E_{2}$. Under this assumption, we can get the following competitive capture systems.

○2014 Wu; licensee Springer. This is an Open Access article distributed under the terms of the Creative Commons Attribution License (http://creativecommons.org/licenses/by/2.0), which permits unrestricted use, distribution, and reproduction in any medium, provided the original work is properly cited. 
The rest of the paper is arranged as follows. We discuss a set of sufficient conditions for the stability of system (1.1) equilibrium based on density dependence in Section 2. It is discussed that system (1.1) stable equilibrium in the optimal acquisition strategy through the extreme value method, by using structuring discrete Hamiltonian function and discrete Pontryagin maximum principle, it is to obtain optimal harvest policy by three equilibriums in Section 3.

\section{Equilibrium and stability}

\subsection{Equilibrium}

By calculating, we can get that system (1.1) has the following equilibriums: $O(0,0)$, $P_{0}\left(\frac{a_{1}-E_{1}}{b_{1}}, 0\right), \bar{P}\left(0, \frac{a_{2}-E_{2}}{c_{2}}\right), P\left(x^{*}, y^{*}\right)$, where

$$
x^{*}=\frac{\left(a_{1}-E_{1}\right) c_{2}-\left(a_{2}-E_{2}\right) c_{1}}{b_{1} c_{2}-b_{2} c_{1}}, \quad y^{*}=\frac{\left(a_{2}-E_{2}\right) b_{1}-\left(a_{1}-E_{1}\right) b_{2}}{b_{1} c_{2}-b_{2} c_{1}} .
$$

Theorem 2.1 $O, P_{0}$ and $\bar{P}$ are non-negative equilibrium points; $P\left(x^{*}, y^{*}\right)$ is a positive equilibrium if and only if

$$
\frac{b_{1}}{b_{2}}>\frac{a_{1}-E_{1}}{a_{2}-E_{2}}>\frac{c_{1}}{c_{2}} \text {. }
$$

\subsection{Stability of the positive equilibrium}

For any initial value $\left(x_{0}, y_{0}\right)$, let $\left\{\left(x_{n}, y_{n}\right)\right\}$ be the solution sequence of system (1.1).

Theorem 2.2 Under the conditions of Theorem 2.1 and further assumption that system (1.1) satisfies the following conditions:

$$
\begin{aligned}
& \text { (1) }\left(1+a_{1}-E_{1}\right)^{2} \leq 4 b_{1} x^{*}, \\
& \text { (2) }\left(1+a_{2}-E_{2}\right)^{2} \leq 4 c_{2} y^{*},
\end{aligned}
$$

the positive equilibrium $P\left(x^{*}, y^{*}\right)$ is locally asymptotically stable in the region $D_{1}=$ $\left\{(x, y) \mid 0<x \leq x^{*}, 0<y \leq y^{*}\right\}$, which is called the attraction domain of the positive equilibrium point $P\left(x^{*}, y^{*}\right)$ in system (1.1).

Proof Let $\left(x_{0}, y_{0}\right) \in D_{1}$, considering the function:

$$
u=b_{1} x^{2}-\left(1+a_{1}-E_{1}\right) x+x^{*}
$$

from condition (1), we have $\Delta_{1}=\left(1+a_{1}-E_{1}\right)^{2}-4 b_{1} x^{*} \leq 0$ and $b_{1}>0$, hence $u \geq 0$.

When $x>0, y>0$, we have

$$
b_{1} x_{n}^{2}+c_{1} x_{n} y_{n}-\left(1+a_{1}-E_{1}\right) x_{n}+x^{*}>0 \text {. }
$$

For $\left(x_{0}, y_{0}\right) \in D_{1}$, according to (1.1) and (2.2), we get

$$
x_{1}=\left(1+a_{1}-E_{1}\right) x_{0}-b_{1} x_{0}^{2}-c_{1} x_{0} y_{0}<x^{*} .
$$


Because $D_{1}$ is in the region surrounded by

$$
a_{1}-E_{1}-b_{1} x_{n}-c_{1} y_{n}=0, \quad a_{2}-E_{2}-b_{2} x_{n}-c_{2} y_{n}=0, \quad x=0, \quad y=0,
$$

it follows that $\Delta x_{0}=x_{0}\left(a_{1}-E_{1}-b_{1} x_{0}-c_{1} y_{0}\right)>0$. That is, $x_{0}<x_{1}$, then $0<x_{0}<x_{1}<x^{*}$.

Similarly, consider the following function:

$$
v=c_{2} y^{2}-\left(1+a_{2}-E_{2}\right) y+y^{*} .
$$

From condition (2) we have

$$
\Delta_{2}=\left(1+a_{2}-E_{2}\right)^{2}-4 c_{2} y^{*} \leq 0
$$

and $c_{2}>0$, so $v \geq 0$. When $x>0, y>0$, we have

$$
b_{2} x_{n} y_{n}+c_{2} y_{n}^{2}-\left(1+a_{2}-E_{2}\right) y_{n}+y^{*}>0 \text {. }
$$

For $\left(x_{0}, y_{0}\right) \in D_{1}$, from (1.1) and (2.3) we get

$$
y_{1}=\left(1+a_{2}-E_{2}\right) y_{0}-b_{2} x_{0} y_{0}-c_{2} y_{0}^{2}<y^{*} .
$$

Because $D_{1}$ is in the region surrounded by

$$
\begin{aligned}
& a_{1}-E_{1}-b_{1} x_{n}-c_{1} y_{n}=0, \quad a_{2}-E_{2}-b_{2} x_{n}-c_{2} y_{n}=0, \\
& y_{n}=0, \quad x=0, \quad y=0,
\end{aligned}
$$

it follows that

$$
\Delta y_{n}=y_{n}\left(a_{2}-E_{2}-b_{2} x_{n}-c_{2} y_{n}\right)>0 .
$$

That is,

$$
y_{1}>y_{0}
$$

thus

$$
0<y_{0}<y_{1}<y^{*}, \quad\left(x_{1}, y_{1}\right) \in D_{1} .
$$

By the recursive method, the solutions $\left(x_{n}, y_{n}\right) \in D_{1}$ of system (1.1) satisfy the conditions of theorem and $0<x_{n}<x_{n+1}<x^{*}, 0<y_{n}<y_{n+1}<y^{*}(n=1,2, \ldots)$.

According to the monotone bounded theorem $\lim _{n \rightarrow \infty} x_{n}=M, \lim _{n \rightarrow \infty} y_{n}=N$.

Let $n \rightarrow \infty$. In (1.1), $\left\{x_{n}\right\},\left\{y_{n}\right\}$ are monotonically increasing sequences and the positive equilibrium point of system (1.1) is unique, we get $M=x^{*}, N=y^{*}$. So the sequence of $\left\{x_{n}\right\}$, $\left\{y_{n}\right\}$ converges to the positive equilibrium $P$. 
Theorem 2.3 Under the conditions of Theorem 2.1 and further assumption that system (1.1) satisfies the following conditions:

(1) $\left(c_{2}+a_{1} c_{2}-E_{1} c_{2}-c_{1} a_{2}+c_{1} E_{2}\right)^{2}>4 b_{1} c_{2}^{2} x^{*}$

(2) $c_{2}+a_{1} c_{2}+c_{1} E_{2} \leq E_{1} c_{2}+c_{1} a_{2}+2 b_{1} c_{2}^{2} x^{*}$

(3) $c_{2}+c_{1} E_{2} \geq a_{1} c_{2}+c_{2} E_{1}+c_{1} a_{2}$

(4) $\left(b_{1}+b_{1} a_{2}-b_{1} E_{2}+a_{1} b_{2}-E_{1} b_{2}\right)^{2}>4 c_{2} b_{1}^{2} y^{*}$

(5) $b_{1}+b_{1} a_{2}+E_{1} b_{2} \leq a_{1} b_{2}+b_{1} E_{2}+2 b_{1} c_{2}^{2} x^{*}$

(6) $b_{1} a_{2}+a_{1} b_{2}+b_{1} E_{2} \leq b_{1}+b_{2} E_{1}$,

$P\left(x^{*}, y^{*}\right)$ in system $(1.1)$ is locally asymptotically stable in the region

$$
D_{2}=\left\{(x, y) \mid x^{*}<x \leq \frac{a_{1}-E_{1}}{b_{1}}, y^{*}<y \leq \frac{a_{2}-E_{2}}{c_{2}}\right\},
$$

which is the attraction domain of $P\left(x^{*}, y^{*}\right)$.

Proof Let $\left(x_{0}, y_{0}\right) \in D_{2}$, since $D_{2}$ is included in the region on the top of the two straight lines $a_{1}-E_{1}-b_{1} x_{n}-c_{1} y_{n}=0, a_{2}-E_{2}-b_{2} x_{n}-c_{2} y_{n}=0$ and $\Delta x_{0}=x_{0}\left(a_{1}-E_{1}-b_{1} x_{0}-c_{1} y_{0}\right)<0$, that is, $x_{1}<x_{0}$.

Consider the following function:

$$
u=b_{1} t^{2}-\left(1+a_{1}-E_{1}-\frac{c_{1} a_{2}-c_{1} E_{2}}{c_{2}}\right) t+x^{*}
$$

From condition (1) we get $\Delta_{1}=\frac{1}{c_{2}^{2}}\left(c_{2}+a_{1} c_{2}-E_{1} c_{2}-c_{1} a_{2}+c_{1} E_{2}\right)^{2}-4 b_{1} x^{*}>0$. This function has two real zero points:

$$
t_{12}=\frac{c_{2}+a_{1} c_{2}-E_{1} c_{2}-c_{1} a_{2}+c_{1} E_{2} \pm \sqrt{\Delta_{1} c_{2}^{2}}}{2 b_{1} c_{2}} .
$$

From condition (2) we get

$$
c_{2}+a_{1} c_{2}-E_{1} c_{2}-c_{1} a_{2}+c_{1} E_{2}-2 b_{1} c_{2}^{2} x^{*} \leq 0 \leq \sqrt{\left(c_{2}+a_{1} c_{2}-c_{1} a_{2}-E_{1} c_{2}\right)^{2}-4 b_{1} c_{2}^{2} x^{*}},
$$

hence $t_{1} \leq x^{*}$.

From condition (3) we get

$$
t_{2}=\frac{c_{2}+a_{1} c_{2}-c_{2} E_{1}-c_{1} a_{2}+c_{1} E_{2}+\sqrt{\Delta_{1} c_{2}^{2}}}{2 b_{1} c_{2}}>\frac{a_{1}-E_{1}}{b_{1}},
$$

$t_{1}<x^{*}<\frac{a_{1}-E_{1}}{b_{1}}<t_{2}$. And when $t_{1}<t<t_{2}, u<0$, so for $x^{*}<t<\frac{a_{1}-E_{1}}{b_{1}}$,

$$
b_{1} t^{2}+\left(\frac{c_{1} a_{2}-c_{1} E_{2}}{c_{2}}-1-a_{1}+E_{1}\right) t+x^{*}<0 .
$$


For $(x, y) \in D_{2}$,

$$
b_{1} x^{2}+c_{1} x y-\left(1+a_{1}-E_{1}\right) x+x^{*}<b_{1} x^{2}+\frac{c_{1}\left(a_{2}-E_{2}\right)}{c_{2}} x-\left(1+a_{1}-E_{1}\right) x+x^{*}<0,
$$

then $x_{1}=\left(1+a_{1}-E_{1}\right) x_{0}-b_{1} x_{0}^{2}-c_{1} x_{0} y_{0}>x^{*}$, hence $x^{*}<x_{1}<x_{0}$.

Consider the auxiliary functions

$$
v=c_{2} s^{2}-\left(1+a_{2}-E_{2}-\frac{a_{1} b_{2}-E_{1} b_{2}}{b_{1}}\right) s+y^{*} .
$$

From conditions (4), (5) and (6), $y^{*}<y_{1}<y_{0}$ can also be proved. From the recursive method available, the solution $\left(x_{n}, y_{n}\right) \in D_{2}$ of system (1.1) satisfies the conditions of theorem, and $x^{*}<x_{n}<x_{n+1}, y^{*}<y_{n}<y_{n+1}(n=1,2, \ldots)$. By the same method used in Theorem 2.2, it can be proved that the solution sequence of system (1.1) converges to the positive equilibrium point $P$.

Based on the actual situation, population $x_{n}>0, y_{n}>0$, then we have the following.

Theorem 2.4 If Theorem 2.2 is satisfied, and system (1.1) satisfies the following conditions:

$$
2 a_{2}-E_{2}<\frac{c_{1} b_{1}+b_{2} c_{2}}{b_{1} c_{2}-b_{2} c_{1}}
$$

then system (1.1) is globally asymptotically stable.

Proof Define a Lyapunov function, $V_{n}\left(x_{n}, y_{n}\right)=b_{2} x_{n}+c_{1} y_{n}$, then

$$
\begin{aligned}
\Delta V_{n}= & b_{2} \Delta x_{n}+c_{1} \Delta y_{n} \\
= & b_{2} x_{n}\left(a_{1}-E_{1}-b_{1} x_{n}-c_{1} y_{n}\right)+c_{1} y_{n}\left(a_{2}-E_{2}-b_{2} x_{n}-c_{2} y_{n}\right) \\
= & b_{2} x_{n}\left(a_{1}-E_{1}\right)-b_{1} b_{2} x_{n}^{2}-c_{1} b_{2} x_{n} y_{n}+c_{1} y_{n}\left(a_{2}-E_{2}\right)-b_{2} c_{1} x_{n} y_{n}-c_{1} c_{2} y_{n}^{2} \\
\leq & b_{2} x_{n}\left(a_{1}-E_{1}\right)-b_{1} b_{2} x_{n}^{2}+c_{1} y_{n}\left(a_{2}-E_{2}\right)-c_{1} c_{2} y_{n}^{2} \\
= & -b_{1} b_{2}\left(x_{n}-\frac{a_{1}-E_{1}}{2 b_{1}}\right)^{2}-c_{1} c_{2}\left(y_{n}-\frac{a_{2}-E_{2}}{2 c_{2}}\right)^{2} \\
& +\frac{b_{2}\left(a_{1}-E_{1}\right)^{2}}{4 b_{1}}+\frac{c_{1}\left(a_{2}-E_{2}\right)^{2}}{4 c_{2}} .
\end{aligned}
$$

From conditions (1) and (2) of Theorem 2.2 we get

$$
\begin{aligned}
& \frac{\left(a_{1}-E_{1}\right)^{2}}{4 b_{1}} \leq x^{*}-\frac{1}{4 b_{1}}-\frac{a_{1}-E_{1}}{2 b_{1}}, \quad \frac{\left(a_{2}-E_{2}\right)^{2}}{4 c_{2}} \leq y^{*}-\frac{1}{4 c_{2}}-\frac{\left(a_{2}-E_{2}\right)}{2 c_{2}}, \\
& \Delta V_{n} \leq-b_{1} b_{2}\left(x_{n}-\frac{a_{1}-E_{1}}{2 b_{1}}\right)^{2}-c_{1} c_{2}\left(y_{n}-\frac{a_{2}-E_{2}}{2 c_{2}}\right)^{2}+b_{2} x^{*}-\frac{b_{2}}{4 b_{1}}-\frac{b_{2}\left(a_{1}-E_{1}\right)}{2 b_{1}} \\
& +c_{1} y^{*}-\frac{c_{1}}{4 c_{2}}-\frac{c_{1}\left(a_{2}-E_{2}\right)}{2 c_{2}} \\
& =-b_{1} b_{2}\left(x_{n}-\frac{a_{1}-E_{1}}{2 b_{1}}\right)^{2}-c_{1} c_{2}\left(y_{n}-\frac{a_{2}-E_{2}}{2 c_{2}}\right)^{2}+\left(a_{2}-E_{2}\right)-\frac{b_{2}}{4 b_{1}}-\frac{b_{2}\left(a_{1}-E_{1}\right)}{2 b_{1}}
\end{aligned}
$$




$$
\begin{aligned}
& -\frac{c_{1}}{4 c_{2}}-\frac{c_{1}\left(a_{2}-E_{2}\right)}{2 c_{2}} \\
= & -b_{1} b_{2}\left(x_{n}-\frac{a_{1}-E_{1}}{2 b_{1}}\right)^{2}-c_{1} c_{2}\left(y_{n}-\frac{a_{2}-E_{2}}{2 c_{2}}\right)^{2} \\
& -\frac{\left(b_{2} c_{2}+b_{1} c_{1}\right)+2\left(a_{2}-E_{2}\right)\left(b_{2} c_{1}-b_{1} c_{2}\right)}{4 b_{1} c_{2}} .
\end{aligned}
$$

From $2\left(a_{2}-E_{2}\right)<\frac{c_{1} b_{1}+b_{2} c_{2}}{b_{1} c_{2}-b_{2} c_{1}}, \Delta V_{n}<0$, then system (1.1) is globally asymptotically stable.

\section{The optimal economic benefit}

As we know, both the fishermen and the fishing companies must consider the costeffectiveness when catching all kinds of fish in terms of the sale price and the capture cost. Suppose that the largest capture intensity is $E_{m}$, then $0<E_{1}+E_{2}=E \leq E_{m}$, the cost is $C$ and the price of the two kinds of group are $p_{1}, p_{2}$. The economic profit is $L=p_{1} E_{1} x+p_{2} E_{2} y-C E$.

For the positive equilibrium point $P\left(x^{*}, y^{*}\right)$, the economic benefits (profits) are

$$
\begin{aligned}
L & =p_{1} E_{1} x^{*}+p_{2} E_{2} y^{*}-C E \\
& =p_{1} E_{1} \frac{\left(a_{1}-E_{1}\right) c_{2}-\left(a_{2}-E_{2}\right) c_{1}}{b_{1} c_{2}-b_{2} c_{1}}+p_{2} E_{2} \frac{\left(a_{2}-E_{2}\right) b_{1}-\left(a_{1}-E_{1}\right) b_{2}}{b_{1} c_{2}-b_{2} c_{1}}-C E \\
& =A\left(E_{1}+B\right)^{2}+D,
\end{aligned}
$$

where

$$
\begin{aligned}
A= & -\frac{p_{1}\left(c_{1}+c_{2}\right)-p_{2}\left(b_{1}+b_{2}\right)}{b_{1} c_{2}-b_{2} c_{1}}, \\
B= & -\frac{p_{1}\left(E c_{1}+a_{1} c_{2}-a_{2} c_{1}\right)+p_{2}\left(E b_{2}+2 E b_{1}+a_{1} b_{2}-a_{2} b_{1}\right)}{2 p_{1}\left(c_{1}+c_{2}\right)-2 p_{2}\left(b_{1}+b_{2}\right)}, \\
D= & \frac{p_{2} E\left(a_{2} b_{1}-a_{1} b_{2}-E b_{1}\right)}{b_{1} c_{2}-b_{2} c_{1}} \\
& -\frac{\left[p_{1}\left(E c_{1}+a_{1} c_{2}-a_{2} c_{1}\right)+p_{2}\left(E b_{2}+2 E b_{1}+a_{1} b_{2}-a_{2} b_{1}\right)\right]^{2}}{4\left(b_{1} c_{2}-b_{2} c_{1}\right)\left[p_{1}\left(c_{1}+c_{2}\right)-p_{2}\left(b_{1}+b_{2}\right)\right]}-C E .
\end{aligned}
$$

Due to the limitation of capture ability $0<E_{1}+E_{2} \leq E_{m}$, from the knowledge of calculus, $A<0$ (that is, $\frac{p_{1}}{p_{2}}>\frac{b_{1}+b_{2}}{c_{1}+c_{2}}$ ), so $L$ has a maximum value.

If $E c_{1}+a_{1} c_{2}>a_{2} c_{1}, 2 E b_{1}+E b_{2}+a_{1} b_{2}>a_{2} b_{1}$, then, when

$$
E_{1}=-B=\frac{p_{1}\left(E c_{1}+a_{1} c_{2}-a_{2} c_{1}\right)+p_{2}\left(E b_{2}+2 E b_{1}+a_{1} b_{2}-a_{2} b_{1}\right)}{2 p_{1}\left(c_{1}+c_{2}\right)+2 p_{2}\left(b_{1}+b_{2}\right)}>0,
$$

$L$ reaches the maximum:

$$
\begin{aligned}
L_{\max }= & D=\frac{p_{2} E\left(a_{2} b_{1}-a_{1} b_{2}-E b_{1}\right)}{b_{1} c_{2}-b_{2} c_{1}} \\
& -\frac{\left[p_{1}\left(E c_{1}+a_{1} c_{2}-a_{2} c_{1}\right)+p_{2}\left(E b_{2}+2 E b_{1}+a_{1} b_{2}-a_{2} b_{1}\right)\right]^{2}}{4\left(b_{1} c_{2}-b_{2} c_{1}\right)\left[p_{1}\left(c_{1}+c_{2}\right)-p_{2}\left(b_{1}+b_{2}\right)\right]}-C E .
\end{aligned}
$$

For the non-negative equilibrium point $\bar{P}\left(0, \frac{a_{2}-E_{2}}{c_{2}}\right)\left(0<E_{2}=E \leq E_{m}\right)$, we obtain the optimal harvest strategy of the non-negative equilibrium point by using the discrete 
Pontryagin maximum principle and the optimal control theory. To obtain the optimal capture, seeking to capture the best efforts of degrees $E_{2}^{*}$, the goal of functions are given:

$$
\bar{L}=\sum_{n=1}^{\infty} \alpha^{n-1}\left(p_{2} y_{n}-C\right) E_{2} \text {. }
$$

According to the discrete maximum principle, seeking optimal control $E_{2}$, the following Hamilton function is introduced:

$$
\bar{H}_{n}=\alpha^{n-1}\left(p_{2} y_{n}-C\right) E_{2}+\lambda_{n}\left(a_{2}+b_{2} x_{n}-c_{2} y_{n}-E_{2}\right) y_{n},
$$

where $\alpha=\frac{1}{1+i}, i$ is the instantaneous discount rate for periods, $\lambda_{n}$ are variables, $E_{2}$ gets maximum value $H_{n}$, which is accompanied by the following equations:

$$
\begin{aligned}
& \Delta \lambda_{n}=\lambda_{n}-\lambda_{n-1}=-\frac{\partial \bar{H}_{n}}{\partial y_{n}}=-\alpha^{n-1} p_{2} E_{2}+c_{2} y_{n} \lambda_{n}, \\
& \Delta^{2} \lambda_{n}=\lambda_{n}-2 \lambda_{n-1}+\lambda_{n-2}=\alpha^{n-2} p_{2} E_{2}(1-\alpha)+c_{2} y_{n}\left(\lambda_{n}-\lambda_{n-1}\right),
\end{aligned}
$$

that is,

$$
\left(1-c_{2} y_{n}\right) \lambda_{n}+\left(c_{2} y_{n}-2\right) \lambda_{n-1}+\lambda_{n-2}=\alpha^{n-2} p_{2} E_{2}(1-\alpha) .
$$

Substituting $n$ into $n-2$ type, we have

$$
\begin{aligned}
& \left(1-c_{2} y_{n}\right) \lambda_{n+2}+\left(c_{2} y_{n}-2\right) \lambda_{n+1}+\lambda_{n}=\alpha^{n} p_{2} E_{2}(1-\alpha), \\
& \Delta=\left(c_{2} y_{n}-2\right)^{2}-4\left(1-c_{2} y_{n}\right)=\left(c_{2} y_{n}\right)^{2}>0 .
\end{aligned}
$$

If $c_{2} y_{n}<1$, we have a solution

$$
\lambda_{n}=-\frac{p_{2} E_{2} \alpha^{n}}{\alpha-1+\alpha c_{2} y_{n}} .
$$

By $\frac{\partial H}{\partial E}=0$, we have

$$
\lambda_{n}=\alpha^{n-1}\left(p_{2} y_{n}-C\right) / y_{n}
$$

because

$$
E_{2}=a_{2}-c_{2} y_{n} .
$$

By (3.6), (3.7) and (3.8), we have

$$
y_{\alpha}=\frac{C(1-\alpha)}{p_{2}(1-\alpha)+\alpha C c_{2}-a_{2} p_{2} \alpha} .
$$

From (3.9), we have $y^{*}=y_{\alpha}$ as the optimal equilibrium solution. So, seeking to capture the best efforts of degrees

$$
E_{2}^{*}=a_{2}-\frac{c_{2} C(1-\alpha)}{p_{2}(1-\alpha)+\alpha C c_{2}-a_{2} p_{2} \alpha},
$$


this is the optimal equilibrium program. Then the economic profit of captured populations is completely controlled by the discount rates $\alpha, C, p_{2}$.

Similarly, consider the non-negative equilibrium point $P_{0}\left(\frac{a_{1}-E_{1}}{b_{1}}, 0\right)\left(0<E_{1}=E \leq E_{m}\right)$. If $b_{1} x_{n}<1$, we have a solution

$$
x_{\alpha}=\frac{C(1-\alpha)}{p_{1}(1-\alpha)+\alpha C b_{1}-a_{1} p_{1} \alpha} .
$$

From (3.10), we have $y^{*}=y_{\alpha}$ as the optimal equilibrium solution. So, seeking to capture the best efforts of degrees

$$
E_{1}^{*}=a_{1}-\frac{b_{1} C(1-\alpha)}{p_{1}(1-\alpha)+\alpha C b_{1}-a_{1} p_{2} \alpha},
$$

this is the optimal equilibrium program. Then the economic profit of captured populations is completely controlled by the discount rates $\alpha, C, p_{1}$.

\section{Conclusion}

This paper qualitatively analyzes a competitive system in situations that are density constrained. We have discussed the stability of equilibrium point in different regions, improved methods of proof in reference. Using the extreme value method to analyze the stable positive equilibrium point is the most optimal way to capture it. By using the Pontryagin maximum principle, through introduces the Hamilton function obtains of the non-negative equilibrium point most superior capture strategy.

\section{Competing interests}

The author declares that they have no competing interests.

\section{Acknowledgements}

This work is supported by the Science and Technology Project of the Education Bureau of Fujian Province (JB13170) and the Start-up Foundation of Science and Technology of Mingjiang Univerity (YKY20132).

Received: 5 April 2013 Accepted: 22 April 2014 Published: 23 Sep 2014

\section{References}

1. Wu, T: Permanence for nonautonomous Lotka-Volterra two species cooperative systems. Bull. Sci. Technol. 25(6), 743-746 (2009)

2. $\mathrm{Wu}, \mathrm{T}$ : Permanence and global stability of a discrete competition feedback-control system with Beddington-DeAngelis functional response. J. Minjiang Univ. Nat. Sci. Ed. 31(2), 16-20 (2010)

3. Wu, T: Permanence of discrete predator-prey system with infinite delay Beddington-DeAngelis functional response J. Minjiang Univ. Nat. Sci. Ed. 31(5), 1-5 (2010)

4. Mao, K, Li, RH: Stability analysis of 2-species competitive model. J. Biomath. 14(3), 288-292 (1999)

5. Wang, J, Zhou, Y: Existence and controllability results for fractional semilinear differential inclusions. Nonlinear Anal., Real World Appl. 12, 3642-3653 (2011)

6. Wang, J, Zhou, Y: Analysis of nonlinear fractional control systems in Banach spaces. Nonlinear Anal. TMA 74(17), 5929-5942 (2011)

7. Wang, J, Zhou, Y: Mittag-Leffler-Ulam stabilities of fractional evolution equations. Appl. Math. Lett. 125, 723-728 (2012)

8. Wang, J, Zhou, Y, Wei, W: Fractional Schrodinger equations with potential and optimal controls. Nonlinear Anal., Real World Appl. 13, 2755-2766 (2012)

9. Wang, J, Zhou, Y, Medved, M: Picard and weakly Picard operators technique for nonlinear differential equations in Banach spaces. J. Math. Anal. Appl. 389, 261-274 (2012)

10. Wang, J, Feckan, M, Zhou, Y: Ulam's type stability of impulsive ordinary differential equations. J. Math. Anal. Appl. 395, 258-264 (2012)

11. Wang, J, Zhou, Y, Feckan, M: Abstract Cauchy problem for fractional differential equations. Nonlinear Dyn. 71, 685-700 (2013)

12. Wang, J, Feckan, M, Zhou, Y: Nonexistence of periodic solutions and asymptotically periodic solutions for fractional differential equations. Commun. Nonlinear Sci. Numer. Simul. 18, 246-256 (2013)

13. Wang, HJ, Feckan, M, Zhou, Y: Relaxed controls for nonlinear fractional impulsive evolution equations. J. Optim. Theory Appl. 156, 13-32 (2013) 
14. Luo, ZX, He, ZR: Analysis of nonlinear fractional control systems in Banach spaces. J. Biomath. 18(3), 269-274 (2003)

15. Lu, HY, Wang, K: Autonomous single-species models and their optimal harvesting policies. J. Syst. Sci. Math. Sci. 24(2), 200-205 (2004)

16. Zhang, Y, Liu, H, Zhang, S, Fan, M, Wang, K: Optimal problem of two species being harvested simultaneously for competing systems. J. Biomath. 13(4), 456-461 (1998)

17. Ganguly, S, Chaudhuri, KS: Regulation of a single-species fishery by taxation. Ecol. Model. 82, 51-60 (1995)

18. Kar, TK: Management of a fishery based on continuous fishing effort. Nonlinear Anal., Real World Appl. 5, 629-644 (2004)

19. Kar, TK: Conservation of a fishery through optimal taxation: a dynamic reaction model. Commun. Nonlinear Sci. Numer. Simul. 10, 121-131 (2005)

20. Yang, XX: Optimal harvest of the prey-predator discrete systems. J. Eng. Math. 21(1), 81-85 (2004)

21. Jiao, JJ, Chen, LS: The extinction threshold on a single population model with pulse input of environmental toxin in a polluted environment. Math. Appl. 22(1), 11-19 (2009)

22. Yang, HX, Sun, Z-Q, Li, YA: Optimal harvest strategy for discrete competing ecosystem. J. Chongqing Inst. Technol. Nat. Sci. Ed. 21(5), 67-70 (2007)

23. Wang, JR, Wei, W, Zhou, Y: Fractional finite time delay evolution systems and optimal controls in infinite dimensional spaces. J. Dyn. Control Syst. 17, 515-535 (2011)

24. Wang, JR, Zhou, Y, Medved, M: On the solvability and optimal controls of fractional integrodifferential evolution systems with infinite delay. J. Optim. Theory Appl. 152, 31-50 (2012)

25. Wang, JR, Zhou, Y, Wei, W: Optimal feedback control for semilinear fractional evolution equations in Banach spaces. Syst. Control Lett. 61, 472-476 (2012)

26. Wang, JR, Zhou, Y: Complete controllability of fractional evolution systems. Commun. Nonlinear Sci. Numer. Simul. $17,4346-4355(2012)$

27. Wang, JR, Fan, ZB, Zhou, Y: Nonlocal controllability of semilinear dynamic systems with fractional derivative in Banach spaces. J. Optim. Theory Appl. 154, 292-302 (2012)

10.1186/1687-1847-2014-241

Cite this article as: Wu: Harvesting analysis of a discrete competitive system. Advances in Difference Equations 2014, 2014:241

\section{Submit your manuscript to a SpringerOpen ${ }^{\circ}$ journal and benefit from:}

- Convenient online submission

Rigorous peer review

- Immediate publication on acceptance

Open access: articles freely available online

- High visibility within the field

- Retaining the copyright to your article 